\title{
Impact Assessment of Poultry Discharge on the Physico-Chemical and Microbiological Water Quality of Olosuru Stream in Ikire, Southwestern Nigeria
}

\author{
Abayomi Tolulope Oyewale ${ }^{*}$, Taiwo Adekanmi Adesakin ${ }^{2}$, Olaoluwa Oyedeji ${ }^{3}$, \\ Adedeji Idowu Aduwo², Mufutau Kolawole Bakare ${ }^{3}$ \\ ${ }^{1}$ Institute of Ecology and Environmental Studies, Faculty of Science, Obafemi Awolowo University, Ile-Ife, Nigeria \\ ${ }^{2}$ Laboratory of Limnology and Hydrobiology, Department of Zoology, Faculty of Science, Obafemi Awolowo University, \\ Ile-Ife, Nigeria \\ ${ }^{3}$ Department of Microbiology, Faculty of Science, Obafemi Awolowo University, Ile-Ife, Nigeria \\ Email: *yomex_01@yahoo.com
}

How to cite this paper: Oyewale, A.T., Adesakin, T.A., Oyedeji, O., Aduwo, A.I. and Bakare, M.K. (2018) Impact Assessment of Poultry Discharge on the Physico-Chemical and Microbiological Water Quality of Olosuru Stream in Ikire, Southwestern Nigeria. Journal of Water Resource and Protection, 10, 1061-1082.

https://doi.org/10.4236/jwarp.2018.1011062

Received: September 19, 2018 Accepted: November 11, 2018 Published: November 14, 2018

Copyright $(9) 2018$ by authors and Scientific Research Publishing Inc. This work is licensed under the Creative Commons Attribution International License (CC BY 4.0).

http://creativecommons.org/licenses/by/4.0/

\section{(c) (i) Open Access}

\begin{abstract}
This study investigated the impact assessment of poultry wastes discharge from a nearby poultry farm on the physico-chemical and microbiological water quality of Olosuru Stream, Ikire, Southwestern Nigeria. Five sampling stations (designated A, B, C, D and E) each located at $250 \mathrm{~m}$ interval along the course of the stream were selected for the study. The physico-chemical parameters in the water body vary in concentration along spatial, monthly and seasonal variation of Olosuru stream. The patterns of spatial distribution of physico-chemical parameters measured for the stream were generally similar except for calcium and hardness which showed significant difference for the five stations. The overall mean values of most of the parameters investigated; $\mathrm{pH}(7.45 \pm 0.24)$, conductivity $(628.69 \pm 255.95 \mu \mathrm{s} / \mathrm{cm})$, TDS $(377.3 \pm 153.55$ $\mathrm{mg} / \mathrm{L})$, sulphate $(10.89 \pm 2.37 \mathrm{mg} / \mathrm{L}), \mathrm{BOD}_{5}(3.19 \pm 2.35 \mathrm{mg} / \mathrm{L})$, cadmium, arsenic, manganese, total heterotrophic bacteria count $(15,080.67 \pm 20,250.67$ $\mathrm{cfu} / \mathrm{ml})$, total coliform bacteria count $(3226 \pm 8426.70 \mathrm{cfu} / \mathrm{ml})$ and total heterotrophic fungi count $(2567.4 \pm 7652.12 \mathrm{cfu} / \mathrm{ml})$ were negatively impacted by poultry wastes dumping into the stream. The concentrations of most parameters exceeded recommended permissible limits of the Nigerian Standard for Drinking Water and World Health Organization for freshwater quality. The water source is therefore deemed not potable and poses hazards to public health if consumed without treatment. There is urgent need for improved management strategies of this water resource for continued sustainability.
\end{abstract}




\section{Keywords}

Microbiological, Physico-Chemical, Pollution, Water Quality, Poultry Waste, Heavy Metals

\section{Introduction}

Water covers about $70 \%$ of Earth's surface, makes up about $70 \%$ of human body mass, and is essential for life [1]. Most plants and animals contain more than $60 \%$ water by volume. The fact that water covers more than two-thirds of the Earth's surface, it is hard to imagine that it is a scarce resource. The problem is that less than $1 \%$ of the water on the planet is readily available for drinking or for most agriculture [2]. Most of the water on Earth, $97 \%$, is salt water stored in the oceans; only $3 \%$ is freshwater [2]. Of all of the freshwater on Earth, $68 \%$ is locked up in the icecaps of Antarctica and Greenland, 30\% is in the ground, and only $0.3 \%$ is contained in surface waters such as lakes, rivers and streams [3]. Water quality reflects the composition of water as affected by natural cause and man's cultural activities expressed in terms of measurable quantities and related to intended water use [4]. Water quality is commonly defined by its physical, chemical, biological and aesthetic (appearance and smell) characteristics [5]. A healthy water environment is that which the water quality supports a rich and varied community of organisms and is conducive to public health. The water quality of a body of water influences its use by the riparian communities for drinking, swimming or commercial purposes. More specifically, water and water bodies may be used by the community for the following purposes: drinking and domestic purposes, recreation (swimming, boating, surfing, skating, etc.), irrigation of crops and watering of livestock, industrial processes, navigation and shipping, production of fin fish and shell fish, protection of aquatic ecosystems, wildlife habitats and scientific study and education [5]. Pollution of the aquatic environment, as defined by [6], occurs when humans introduce, either by direct discharge to water or indirectly (for example through atmospheric pollution or water management practices), substances or energy that result in deleterious effects such as: hazards to human health, harm to living resources, hindrance to aquatic activities such as fishing and impairment of water quality with respect to its use in economic activities, or reduction of amenity value.

It is estimated that production and consumption of poultry meat in developing countries will increase by 3.6 percent and 3.5 percent, respectively, per annum from 2005 to 2030 because of rising incomes, diversification of diets and expanding markets [7]. There is little doubt that the consumption and, thus, production of poultry will continue to increase relative to the world's population and economy. Consequently, environmental parameters impacted by waste by-products resulting from the production and processing of poultry products are of increasing importance worldwide. The increase in concentration of livestock and poultry also leads to increased concentration of animal manure that must be manage. As production has shifted to much larger, more concentrated 
operations, livestock and poultry operations have become separate from the land base that produces their feed [8]. While livestock manure can be a resource, it can also degrade environmental quality, particularly surface and ground water if not managed appropriately [9]. Impacts of poultry litter application on surface runoff and chemical water quality in receiving streams were reported in [10] [11] [12]. Most outbreaks of waterborne and foodborne gastrointestinal illness, even those caused by zoonotic pathogens, are attributable to human faecal contamination, although agricultural sources have been implicated in a number of cases [13].

Olosuru stream is a perennial surface water source situated within Ikire Township. The water serves both domestic and agricultural purposes to the riparian users within the town. However, there is scarcity of information on the operations of a poultry farm situated along the course of the stream water source. This study aimed to investigate the impact of the activities of this poultry farm, with a view to determining the potability of the stream water for its primary (domestic and agricultural) purposes.

\section{Materials and Methods}

\subsection{Study Area}

The study was based along the course of Olosuru Stream, Ikire urban Area in Osun State, Nigeria. Five sampling points (designated A, B, C, D and E) each located at $250 \mathrm{~m}$ interval along the course of the stream were selected for the study. Points A and B are before the discharge point (upstream), C is point of discharge, while $\mathrm{D}$ and $\mathrm{E}$ are after point of discharge (downstream) as shown in Figure 1. The grid co-ordinates of each point were determined using portable Global Positioning Systems (GPS) equipment (Model GERMIN GPS map 76CSX). The study area lies within latitude $07^{\circ} 20^{\prime}$ and $07^{\circ} 22^{\prime} \mathrm{N}$ and longitude $004^{\circ} 10^{\prime}$ and $004^{\circ} 11^{\prime} \mathrm{E}$. Ikire urban area is the headquarters of Irewole Local Government Area of Osun State. The Local Government Area is bounded in the north by Ayedire, in the south by Isokan, in the east by Ayedaade, and in the south east by Ife-North Local Government Areas of Osun state respectively. It also shares boundary with Egbeda Local Government Area of Oyo state to the west. It has a land mass of $271 \mathrm{~km}^{2}$. According to the 2006 census by the National Population Council [14], Ikire has an estimated population of 143,599 . The climate is humid tropical type with a mean annual temperature of about $27^{\circ} \mathrm{C}$ and a mean annual rainfall of over $1300 \mathrm{~mm}$. The geology survey map suggests that the basement complex in this area comprises migmatised gneisses and granite. There are occurrences of schist and quartzite, occasionally amphibolite, gabro, diorites; the dominant in the surveyed area is gneisses [15] [16]. The soils are mainly the well-drained Apomu series known as Cambic Arenosols [17].

\subsection{Sample Collection}

Water samples were collected into four sets of sterilized, sealed and well-labelled sample bottles (namely Biological Oxygen Demand (BOD) bottles, Dissolved 


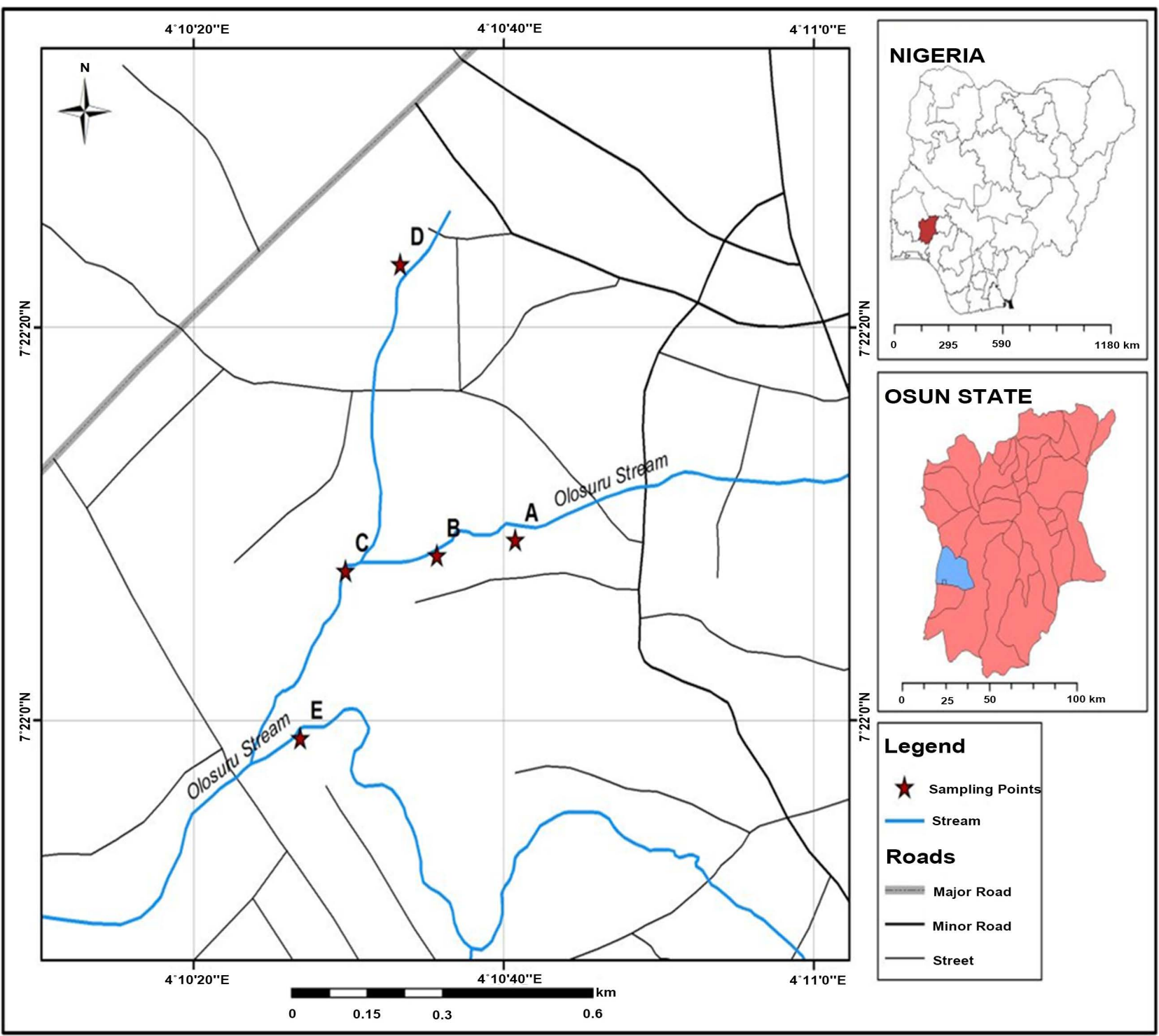

Figure 1. Map showing the sampling points of Olosuru stream, Ikire, Southwestern Nigeria.

Oxygen (DO) bottles, sterile universal bottles and sterile plastic bottles) for BOD analysis, DO analysis, microbiological analysis and physico-chemical analysis respectively. At the sampling points, the sampling bottles and caps were rinsed three times with the water to be sampled prior to sampling. Water samples were then collected directly into the sampling bottles against the run of flow of the stream with standard procedures well observed [18]. Water sampling was conducted six times spanning through the rain (September, October 2014 and April 2015) and dry seasons (November 2014, January and February 2015).

\subsection{Water Analysis}

Physico-Chemical Parameters and Microbial Analysis

The stream water samples were examined for physico-chemical parameters such as ambient air and water temperature using mercury-in-glass bulb thermometer 
on the spot of collection; apparent colour, true colour and turbidity were determined through turbidimetric method using (JENWAY 6051) colorimeter; Total Suspended Solids, Total Dissolved Solids and Total Solids were analyzed by gravimetric method. Conductivity and $\mathrm{pH}$ were analyzed using Conductivity and $\mathrm{pH}$ meter respectively (PCE-PHD 1), calcium and magnesium were determined through complexometric titration; sulphate was analyzed using turbidimetric method; nitrate-nitrogen was determined through instrumental method using ultra violet visible spectrophotometer (Thermo Scientific). Dissolved Oxygen and Biological Oxygen Demand $\left(\mathrm{BOD}_{5}\right)$ were analyzed through Iodometric titration using Winkler's reagent, sulphuric acid, starch indicator and sodium thiosulphate. Heavy metals (cadmium, arsenic, iron, manganese and lead) were analyzed using Atomic Absorption Spectrophotometer (PG 990). The microbiological parameters determined included total heterotrophic bacteria count, total coliform bacteria count and total fungi count, using serial dilution method and pour plate techniques. Streaking method was used to obtained pure bacterial isolates by sub-culturing a previously incubated plate onto a freshly prepared sterile plate while pure fungi isolates were obtained using cutting technique by sub-culturing a previously incubated plate onto a freshly prepared sterile plate. The water samples were analyzed with the holding time of the respective parameters using standard methods with adequate quality control measures. The bacterial isolates were characterized using colonial, morphological and biochemical identification methods. They were further identified using Bergey's manual of Determinative Bacteriology; the microscopic and macroscopic identification of fungi isolates were carried out using standard methods.

\subsection{Data Analysis}

The data obtained were analyzed using appropriate statistical methods including descriptive statistics, inferential statistics (ANOVA, regression, correlation analyses) through Statistic Package for Social Science (SPSS) version 23 and PAST (Paleontological Statistics) statistical software.

\section{Results}

The results of the physico-chemical characteristics, microbial loads and some heavy metals of water from Olosuru stream at the different sampling stations are summarized in Tables 1-5 showed the identifications of bacterial and fungal isolates from the water samples respectively. Table 6 presented the Principal component analysis showing the spatial and seasonal variation of physico-chemical parameters and microbial loads of Olosuru stream, Ikire, Southwestern, Nigeria.

Table 1 shows the results of the physico-chemical parameters measured for seasonal variations. Air temperature ranged from $25.00^{\circ} \mathrm{C}$ to $29.50^{\circ} \mathrm{C}$ with a mean value of $26.68^{\circ} \mathrm{C} \pm 1.17^{\circ} \mathrm{C}$. The mean air temperature recorded during the wet season $\left(26.9^{\circ} \mathrm{C} \pm 1.48^{\circ} \mathrm{C}\right)$ was higher than value observed for the dry season 
Table 1. Seasonal variation for physico-chemical parameters and microbial loads of Olosuru stream, Ikire, Southwestern Nigeria.

\begin{tabular}{|c|c|c|c|c|c|c|c|c|}
\hline \multirow{2}{*}{ Parameter } & \multicolumn{2}{|c|}{ Dry season } & \multicolumn{2}{|c|}{ Wet season } & \multicolumn{2}{|c|}{ Anova } & \multicolumn{2}{|c|}{ Overall } \\
\hline & Min - Max & Mean \pm SD & Min - Max & Mean \pm SD & & & Min - Max & Mean \pm SD \\
\hline Air temp $\left({ }^{\circ} \mathrm{C}\right)$ & $25-28$ & $26.45 \pm 0.75$ & $25-29.50$ & $26.9 \pm 1.48$ & 1.108 & 0.301 & $25-29.50$ & $26.68 \pm 1.17$ \\
\hline Water temp $\left({ }^{\circ} \mathrm{C}\right)$ & $25.5-27.50$ & $26.45 \pm 0.64$ & $25-29.0$ & $26.68 \pm 1.61$ & 0.272 & 0.606 & $25-29.0$ & $26.56 \pm 1.21$ \\
\hline True colour (Pt.CO.) & $93.79-101.29$ & $98.14 \pm 2.60$ & $94.29-101.39$ & $99.06 \pm 2.06$ & 1.137 & 0.295 & $93.79-101.39$ & $98.6 \pm 2.35$ \\
\hline Turbidity (NTU) & $0.37-91.84$ & $21.27 \pm 22.46$ & $3.63-88.57$ & $21.49 \pm 20.73$ & 0.001 & 0.978 & $0.37-91.84$ & $21.38 \pm 21.24$ \\
\hline TS (mg/L) & $214-1398$ & $970.4 \pm 421.98$ & $249-1801$ & $1269.6 \pm 499.51$ & 3.141 & 0.087 & $214-1801$ & $1120 \pm 479.13$ \\
\hline TSS(mg/L) & $17-972$ & $599.93 \pm 394.71$ & $16-1343$ & $819.93 \pm 447.43$ & 2.039 & 0.164 & $16-1343$ & $709.93 \pm 429.39$ \\
\hline Conductivity $(\mu \mathrm{s} / \mathrm{cm})$ & $328.34-1546.98$ & $617.41 \pm 296.23$ & $270.01-111.62$ & $639.98 \pm 218.37$ & 0.056 & 0.814 & $270.01-1546.98$ & $628.69 \pm 255.95$ \\
\hline TDS (mg/L) & $197-928$ & $370.47 \pm 177.69$ & $162-667$ & $384.13 \pm 131.01$ & 0.057 & 0.812 & $162-928$ & $377.3 \pm 153.55$ \\
\hline $\begin{array}{c}\text { Hardness } \\
\left(\mathrm{CaCO}_{3} \mathrm{mg} / \mathrm{L}\right)\end{array}$ & $57.108-177.30$ & $114.53 \pm 27.51$ & $71.07-181.94$ & $127.43 \pm 27.43$ & 1.655 & 0.209 & $57.108-181.94$ & $120.98 \pm 27.78$ \\
\hline $\mathrm{DO}(\mathrm{mg} / \mathrm{L})$ & $1.2-7.0$ & $3.95 \pm 2.22$ & $1.4-7.80$ & $4.05 \pm 2.05$ & 0.018 & 0.896 & $1.2-7.80$ & $4.00 \pm 2.10$ \\
\hline $\mathrm{BOD}(\mathrm{mg} / \mathrm{L})$ & $0.5-8.8$ & $3.64 \pm 2.60$ & $0.4-7.50$ & $2.73 \pm 2.06$ & 1.137 & 0.295 & $0.4-8.0$ & $3.19 \pm 2.35$ \\
\hline Calcium (mg/L) & $18.66-66.24$ & $41.86 \pm 10.75$ & $23.26-70.08$ & $46.56 \pm 11.05$ & 1.396 & 0.247 & $18.66-70.08$ & $44.21 \pm 10.98$ \\
\hline Magnesium (mg/L) & $0.28-11.44$ & $4.71 \pm 2.995$ & $0.86-10.27$ & $5.25 \pm 3.05$ & 0.249 & 0.622 & $0.28-11.44$ & $4.98 \pm 2.96$ \\
\hline Sulphate (mg/L) & $4.1-13.69$ & $10.39 \pm 2.47$ & $8.16-15.17$ & $11,382.23 \pm 2.23$ & 1.316 & 0.261 & $4.1-15.17$ & $10.89 \pm 2.37$ \\
\hline $\begin{array}{l}\text { Nitrate-nitrogen } \\
(\mathrm{mg} / \mathrm{L})\end{array}$ & $3.67-16.09$ & $11.29 \pm 3.45$ & $6.22-15.26$ & $10.88 \pm 2.80$ & 0.136 & 0.715 & $3.67-16.09$ & $11.09 \pm 3.10$ \\
\hline As $(\mathrm{mg} / \mathrm{L})$ & $0.01-0.02$ & $0.01 \pm 0.00$ & $0.003-0.02$ & $0.01 \pm 0.01$ & 0.009 & 0.927 & $0.00-0.02$ & $0.01 \pm 0.00$ \\
\hline $\mathrm{Fe}(\mathrm{mg} / \mathrm{L})$ & $0.09-0.27$ & $0.19 \pm 0.02$ & $0.104-0.31$ & $0.22 \pm 0.06$ & 0.682 & 0.416 & $0.09-0.31$ & $0.21 \pm 0.06$ \\
\hline $\mathrm{Mn}(\mathrm{mg} / \mathrm{L})$ & $0.04-0.10$ & $0.06 \pm 0.03$ & $0.04-0.11$ & $0.07 \pm 0.29$ & 0.615 & 0.440 & $0.04-0.11$ & $0.07 \pm 0.03$ \\
\hline $\mathrm{Pb}(\mathrm{mg} / \mathrm{L})$ & $0.01-0.02$ & $0.01 \pm 0.00$ & $0.01-0.03$ & $0.018 \pm 0.06$ & 12.174 & 0.002 & $0.01-0.03$ & $0.02 \pm 0.01$ \\
\hline THBC (cfu/ml) & $310-68,100$ & $20,202.67 \pm 21,808.05$ & $320-58,100$ & $9958.67 \pm 17,823.10$ & 1.984 & 0.170 & $310-68,100$ & $15,080.67 \pm 20,250.67$ \\
\hline TCBC (cfu/ml) & $0-38,000$ & $3305.33 \pm 9754.45$ & $0-24,100$ & $3146.67 \pm 7206.10$ & 0.003 & 0.960 & $0-38,000$ & $3226 \pm 8426.70$ \\
\hline THFC (sfu/ml) & $0-31,000$ & $4914.13 \pm 10,462.54$ & $0-390$ & $220.67 \pm 162.72$ & 3.018 & 0.093 & $0-31,000$ & $2567.4 \pm 7652.12$ \\
\hline
\end{tabular}

$\left(26.45^{\circ} \mathrm{C} \pm 0.75^{\circ} \mathrm{C}\right)$. The overall mean water temperature for the study period was $26.56^{\circ} \mathrm{C} \pm 1.21^{\circ} \mathrm{C}$ while mean water temperature for the dry season $\left(26.45^{\circ} \mathrm{C}\right.$ $\left.\pm 0.64^{\circ} \mathrm{C}\right)$ was slightly lower than the wet season value $\left(26.68^{\circ} \mathrm{C} \pm 1.61^{\circ} \mathrm{C}\right)$. Higher mean values for TS, TSS, True colour $(1269.6 \pm 499.51 \mathrm{mg} / \mathrm{L}, 819.93 \pm$ $447.43 \mathrm{mg} / \mathrm{L}$ and $99.06 \pm 2.06 \mathrm{Pt}$.Co.) were recorded during the wet season than dry season $(970.4 \pm 421.98 \mathrm{mg} / \mathrm{L}, 599.93 \pm 394.71 \mathrm{mg} / \mathrm{L}, 98.14 \pm 2.60$ Pt.Co. $)$ while maximum mean value was observed for apparent colour $(91.19 \pm 2.51$ Pt.Co.) during the dry season than wet season (90.19 \pm 2.28 Pt.Co.). Turbidity ranged from 3.63 to $88.57 \mathrm{NTU}$ during the wet season and 0.37 to $91.84 \mathrm{NTU}$ during the dry season. Low mean concentrations of dissolved oxygen (DO) were generally observed during the dry season $(3.95 \pm 2.22 \mathrm{mg} / \mathrm{L})$ while high 


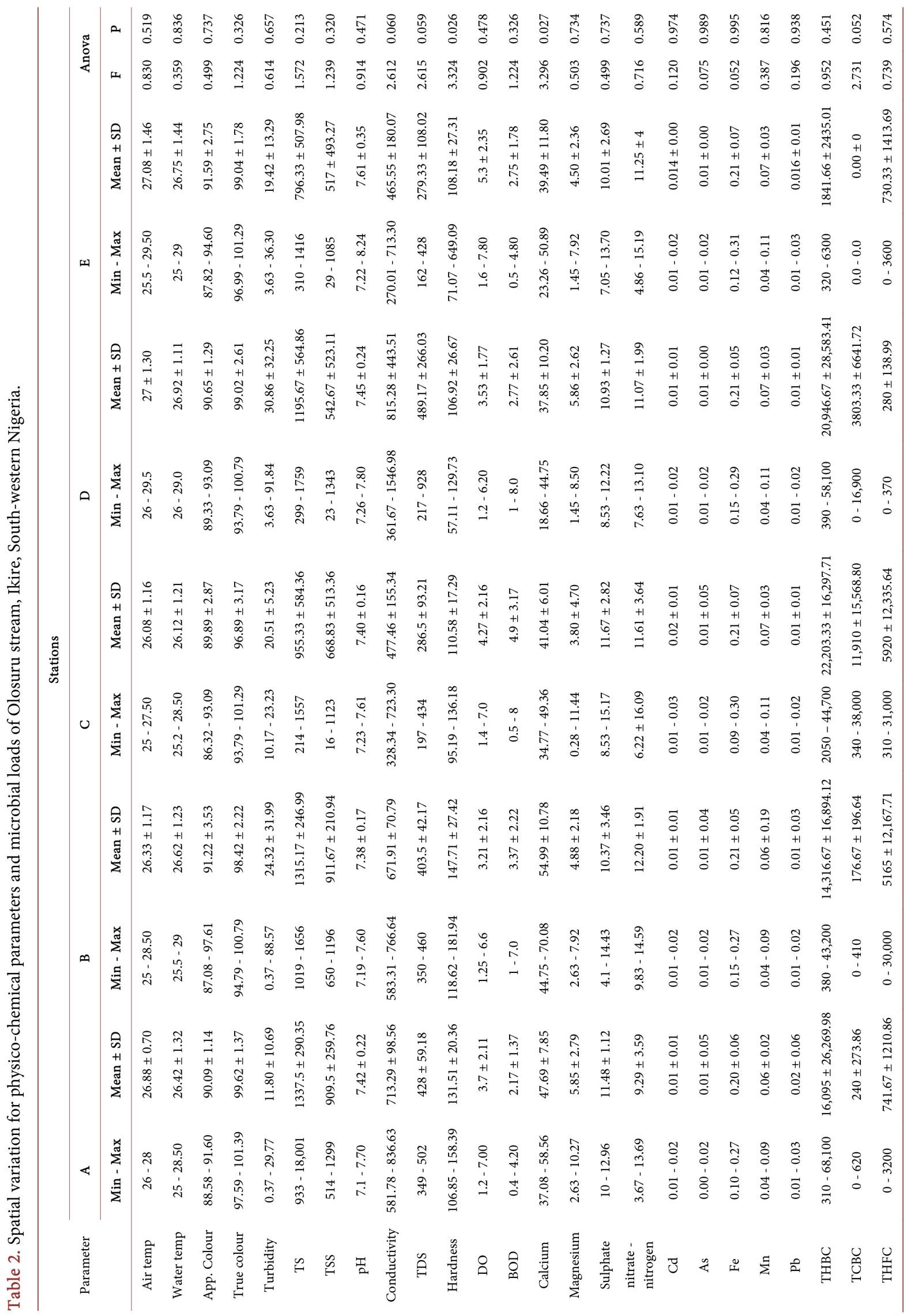




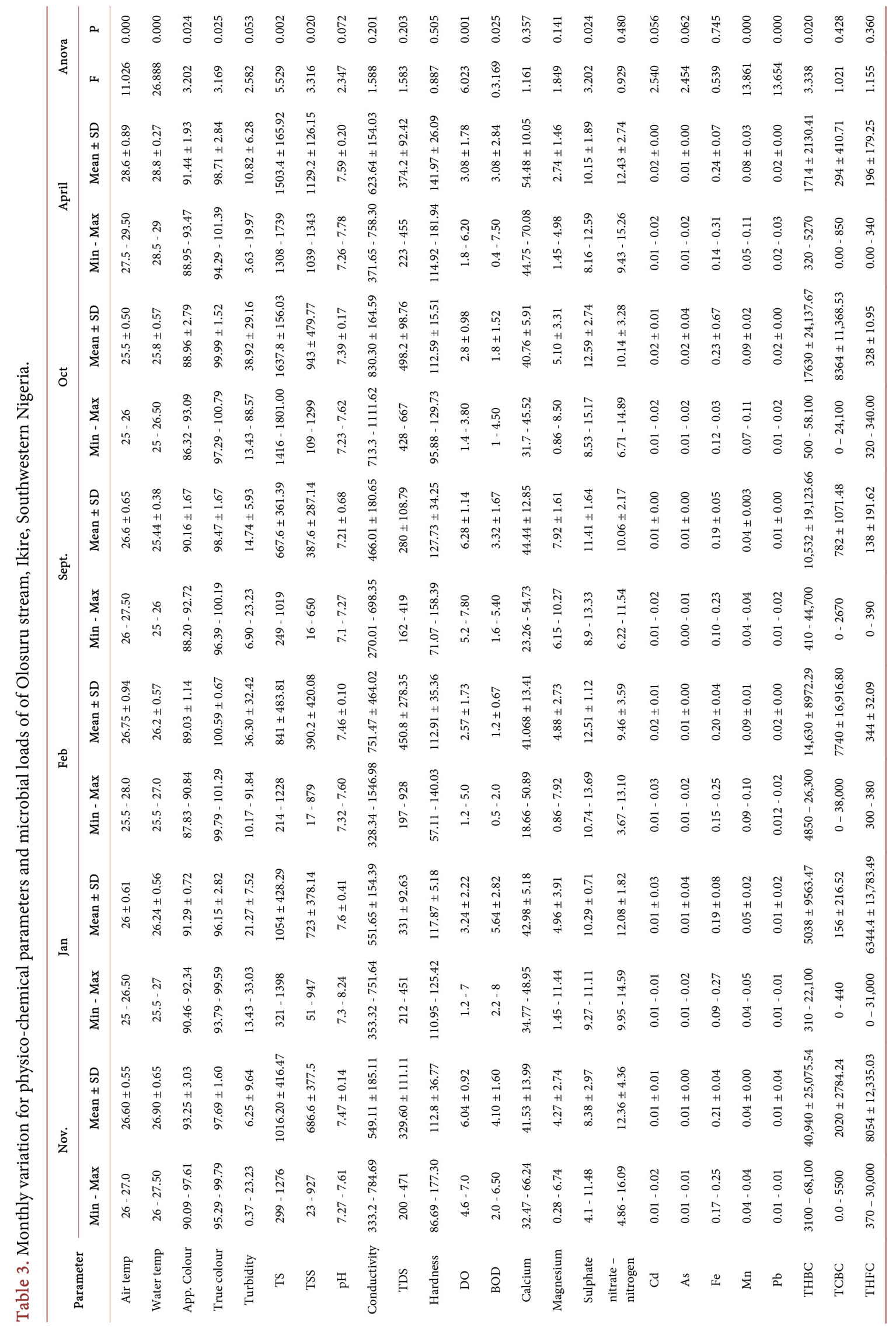


Table 4. Biochemical characterizations of bacterial isolates from olosuru stream in ikire.

\begin{tabular}{|c|c|c|c|c|c|c|c|c|c|c|c|c|c|c|c|c|c|c|c|}
\hline \multirow{2}{*}{ Biochemicals } & \multicolumn{19}{|c|}{ Isolate code } \\
\hline & 1 & 2 & 3 & 4 & 5 & 6 & 7 & 8 & 9 & 10 & 11 & 12 & 13 & 14 & 15 & 16 & 17 & 18 & 19 \\
\hline Gram's Staining & + & + & + & + & - & - & - & - & - & + & + & - & - & - & + & + & - & - & + \\
\hline Spore Staining & - & - & - & + & NA & NA & NA & NA & NA & + & + & + & + & + & + & - & + & NA & - \\
\hline Catalase & + & + & - & - & + & + & + & + & + & + & + & - & + & + & + & + & + & + & + \\
\hline Oxidase & NA & NA & NA & NA & + & + & + & - & - & NA & + & - & - & + & - & - & - & + & - \\
\hline Starch Hydrolysis & - & + & - & + & - & NA & NA & NA & NA & - & + & + & + & NA & NA & NA & + & NA & NA \\
\hline Citrate & - & + & + & - & - & - & + & - & - & + & - & - & - & + & + & + & + & - & - \\
\hline Mannitol & - & A & - & - & - & - & - & AG & A & A & - & AG & AG & A & - & AG & - & - & AG \\
\hline Glucose & - & - & A & A & AG & A & - & - & AG & - & A & A & AG & A & AG & AG & A & - & AG \\
\hline Lactose & NA & NA & - & NA & NA & A & A & - & - & NA & - & A & - & A & NA & NA & A & - & - \\
\hline Arabinose & NA & NA & NA & NA & NA & NA & - & NA & NA & NA & NA & NA & NA & NA & NA & NA & NA & NA & NA \\
\hline $6.5 \% \mathrm{NaCl}$ & - & + & + & - & + & - & - & NA & NA & - & - & + & + & + & + & + & + & NA & + \\
\hline Methyl Red Test & - & - & + & + & - & - & - & + & + & - & + & + & + & - & - & + & - & + & + \\
\hline $\begin{array}{c}\text { Voges-Proskaur } \\
\text { Test }\end{array}$ & - & + & - & - & + & - & + & + & - & - & - & - & - & + & + & + & - & + & - \\
\hline Motility & NA & NA & NA & NA & NA & + & NA & NA & - & NA & NA & + & + & + & NA & - & + & - & NA \\
\hline Indole & NA & NA & NA & NA & + & - & - & - & + & NA & NA & + & - & - & NA & NA & - & NA & NA \\
\hline Nitrate & NA & NA & NA & NA & + & - & + & + & + & - & NA & + & + & + & NA & NA & + & - & NA \\
\hline Urease & NA & NA & NA & NA & + & + & - & + & + & NA & - & - & + & NA & NA & NA & + & + & + \\
\hline Hydrogen Sulphide & NA & NA & NA & NA & NA & + & - & - & - & NA & - & NA & - & - & NA & NA & NA & - & - \\
\hline 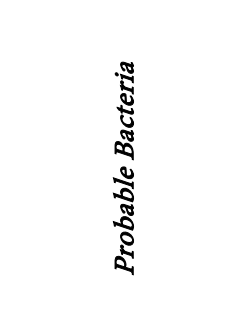 & 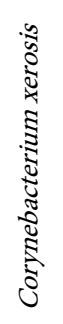 & 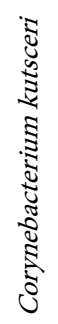 & 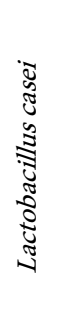 & 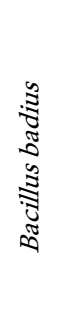 & 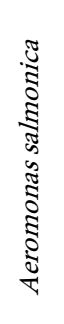 & 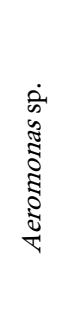 & 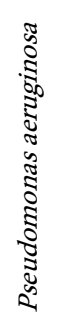 & 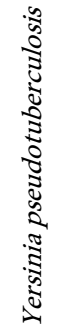 & 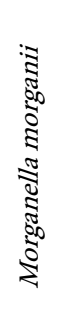 & 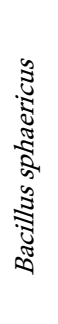 & 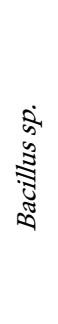 & 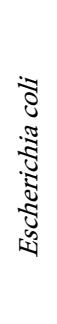 & 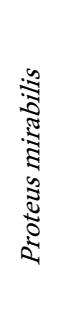 & $\begin{array}{l}\dot{2} \\
\dot{0} \\
\stackrel{0}{0} \\
\dot{1}\end{array}$ & 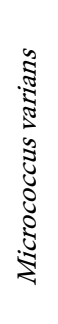 & 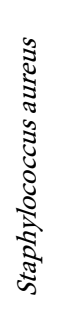 & 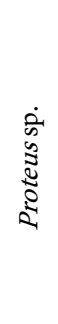 & 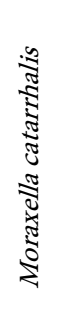 & 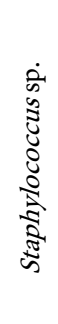 \\
\hline
\end{tabular}

Key: AG = Production of Gas and Acid, A = Production of Gas, $+=$ Positive test, $-=$ Negative test, NA = Not Applicable .

concentrations of DO were observed during the wet season $(4.05 \pm 2.05 \mathrm{mg} / \mathrm{L})$. An overall BOD mean which ranged from 0.4 to $8.0 \mathrm{mg} / \mathrm{L}$, with mean concentration of $3.19 \pm 2.35 \mathrm{mg} / \mathrm{L}$ was observed for BOD in this study. The BOD mean concentration recorded was higher during the dry seasons than wet season. Hydrogen ion concentration $(\mathrm{pH})$ ranged from 7.1 to 8.24 with overall mean of 7.45 \pm 0.24 . The mean $\mathrm{pH}$ for the dry season $(7.51 \pm 0.25)$ was higher than the dry season $(7.39 \pm 0.22)$. The higher mean concentration for conductivity, TDS, calcium, magnesium and Hardness $(639.98 \pm 218.37 \mu \mathrm{s} / \mathrm{cm}, 384.13 \pm 131.01 \mathrm{mg} / \mathrm{L}$, $46.56 \pm 11.05 \mathrm{mg} / \mathrm{L}, 5.25 \pm 3.05 \mathrm{mg} / \mathrm{L}$ and $127.43 \pm 27.43 \mathrm{CaCO}_{3} \mathrm{mg} / \mathrm{L}$ ) were recorded during the wet season than dry season. Overall Nitrate-nitrogen 
Table 5. Macroscopic and microscopic characteristics of fungi isolated from olosuru stream water samples in ikire township.

\begin{tabular}{|c|c|c|c|}
\hline$s / n$ & Fungi & Macroscopic feature & Microscopic feature \\
\hline 1 & Aspergillus brevipes & Greyish green & $\begin{array}{l}\text { Conidiophores hyaline, simple, ellipsoidally at the apex, bearing } \\
\text { spore heads composed of catenulate conidia. }\end{array}$ \\
\hline 2 & Aspergillus flavus & $\begin{array}{l}\text { Lime green } \\
\text { Texture is woolly to cotton }\end{array}$ & $\begin{array}{l}\text { Conidiophore upright, simple terminating in a globose or clavate } \\
\text { swelling, bearing phialides at the apex or radiating from the entire } \\
\text { surface. }\end{array}$ \\
\hline 3 & Aspergillus fumigatus & Light green & $\begin{array}{l}\text { Conidiophore hyaline, simple, thin in cell wall, inflated clavately } \\
\text { at the apex forming often nodded vesicles, bearing conidial heads. }\end{array}$ \\
\hline 4 & Aspergillus niger & $\begin{array}{l}\text { Initially white, quickly becoming black } \\
\text { with conidial production }\end{array}$ & $\begin{array}{l}\text { Conidiophore hyaline or pale brown, erect, simple, thick-walled, } \\
\text { with foot cells basally. }\end{array}$ \\
\hline 5 & Aspergillus parasiticus & Dark yellowish-green and velvety & $\begin{array}{l}\text { Conidiophores erect, simple, rough in the surface, with foot cells } \\
\text { basally inflated at the apex. }\end{array}$ \\
\hline 6 & Mucor circinellioides & Yellowish-brown & $\begin{array}{l}\text { Sporangiospores hyaline, simple, sporangium and } \\
\text { chlamydospores produced singly and in short chains. }\end{array}$ \\
\hline 7 & Mucor luteus & $\begin{array}{l}\text { Initially white, turns to greyish brown } \\
\text { with ageing }\end{array}$ & $\begin{array}{l}\text { Sporangiophore and sporangium with collar, columella and } \\
\text { sporangiospores. }\end{array}$ \\
\hline 8 & Neurospora sp. & White colouration, rapid growth & $\begin{array}{l}\text { Ascospores hyaline, simple, ellipsoidal, nearly smooth, germ } \\
\text { pores disposed at the ends of the ascospores. }\end{array}$ \\
\hline 9 & Rhizoctonia sp. & Deep brown & $\begin{array}{l}\text { Club-shaped basidia with multiple apical sterigmata, oval, hyaline } \\
\text { basidiospores. }\end{array}$ \\
\hline 10 & Rhizopus sp. & $\begin{array}{l}\text { Greyish white } \\
\text { Texture is cotton-candy like }\end{array}$ & $\begin{array}{l}\text { Sporangiospores hyaline, simple, smooth in the surface, } \\
\text { Sporangia are round with flattened bases, located at the tip of the } \\
\text { sporangiophores. }\end{array}$ \\
\hline 11 & Rhizopus stolonifer & Rapid growth with white colouration & Ellipsoidal structure with rhizoid may be immature zygospore. \\
\hline 12 & Trichoderma harzianum & Green colouration & $\begin{array}{l}\text { Conidiophores hyaline, branched, and demonstrate a pyramidal } \\
\text { arrangement; Conidia are unicellular, ellipsoidal, green in color, } \\
\text { smooth walled. }\end{array}$ \\
\hline
\end{tabular}

$\left(\mathrm{NO}_{3}-\mathrm{N}\right)$ values ranged from 3.67 to $16.09 \mathrm{mg} / \mathrm{L}$ while mean concentration obtained during the dry season $(11.29 \pm 3.45)$ is higher than wet season $(10.88 \pm$ 2.80). Concentrations of lead ranged from 0.01 to $0.03 \mathrm{mg} / \mathrm{L}$ in samples collected during the wet season and from 0.01 to $0.02 \mathrm{mg} / \mathrm{L}$ during the dry season. Higher mean concentration were recorded for $\mathrm{Cd}, \mathrm{Fe}, \mathrm{Mn}$ and $\mathrm{Pb}(0.02 \pm 0.00 \mathrm{mg} / \mathrm{L}$, $0.22 \pm 0.06 \mathrm{mg} / \mathrm{L}, 0.07 \pm 0.29 \mathrm{mg} / \mathrm{L}$ and $0.018 \pm 0.06 \mathrm{mg} / \mathrm{L}$ ) during wet season than dry season. The recorded total heterotrophic bacterial counts (THBC) from the stream water samples fell within the range of $3.10 \times 10^{1}-6.81 \times 10^{4}$ $\mathrm{CFU} \cdot \mathrm{mL}^{-1}$ with an overall mean of $1.51 \times 10^{4} \pm 2.02 \times 10^{4} \mathrm{CFU} \cdot \mathrm{mL}^{-1}$. The total coliform bacteria counts (TCBC) over the period of study were in the range of 0 $-3.8 \times 10^{4} \mathrm{CFU} \cdot \mathrm{mL}^{-1}$ with an overall mean of $\left(3.23 \times 10^{3} \pm 8.43 \times 10^{3} \mathrm{CFU} \cdot \mathrm{mL}^{-1}\right)$ while the overall mean value for total heterotrophic fungi count (THF) over the study period was $\left(2.57 \times 10^{3} \pm 7.65 \times 10^{3} \mathrm{SFU} \cdot \mathrm{mL}^{-1}\right)$ within the range of 0.00 $3.10 \times 10^{4} \mathrm{SFU} \cdot \mathrm{mL}^{-1}$.

Table 2 shows the results of the physico-chemical parameters measured for spatial variations. The lowest mean air temperature $\left(26.80^{\circ} \mathrm{C} \pm 1.16^{\circ} \mathrm{C}\right)$ was recorded in station $\mathrm{C}$ while the highest air temperature $\left(27.08^{\circ} \mathrm{C} \pm 1.46^{\circ} \mathrm{C}\right)$ was 
Table 6. Principal component analysis showing the spatial and seasonal variation of physico-chemical parameters and microbial loads of Olosuru stream, Ikire, Southwestern, Nigeria.

\begin{tabular}{|c|c|c|c|c|c|c|c|c|c|c|c|c|c|c|}
\hline & \multicolumn{10}{|c|}{ Spatial variation } & \multicolumn{4}{|c|}{ Seasonal variation } \\
\hline & \multicolumn{2}{|c|}{ Station A } & \multicolumn{2}{|c|}{ Station B } & \multicolumn{2}{|c|}{ Station C } & \multicolumn{2}{|c|}{ Station D } & \multicolumn{2}{|c|}{ Station E } & \multicolumn{2}{|c|}{ Wet season } & \multicolumn{2}{|c|}{ Dry season } \\
\hline & APCA & APCA & BPCA & BPCA & CPCA & CPCA & DPCA & DPCA & EPCA & EPCA & WSPAC & WSPCA & DSPCA & DSPCA \\
\hline & 1 & 2 & 1 & 2 & 1 & 2 & 1 & 2 & 1 & 2 & 1 & 2 & 1 & 2 \\
\hline Eigenvalue & 4.80 & 1.14 & 3.88 & 1.81 & 3.87 & 1.50 & 4.67 & 1.23 & 3.86 & 1.31 & 10.38 & 3.37 & 10.15 & 2.56 \\
\hline$\%$ variance & 79.94 & 19.03 & 64.72 & 30.15 & 64.55 & 24.94 & 77.84 & 20.54 & 64.37 & 21.83 & 69.18 & 22.46 & 67.68 & 17.05 \\
\hline $\begin{array}{l}\text { Cumulative } \\
\text { variance }\end{array}$ & 79.94 & 98.97 & 64.72 & 94.87 & 64.55 & 89.49 & 77.84 & 98.38 & 64.37 & 86.20 & 69.18 & 91.64 & 67.68 & 84.73 \\
\hline Air temp & -0.99 & -0.38 & -0.90 & -0.44 & -0.75 & -0.11 & -0.94 & -0.41 & -1.14 & 0.06 & -1.37 & -0.34 & -1.16 & -0.52 \\
\hline Water temp & -1.00 & -0.38 & -0.90 & -0.44 & -0.75 & -0.11 & -0.95 & -0.42 & -1.14 & 0.06 & -1.37 & -0.34 & -1.16 & -0.52 \\
\hline App. Colour & -0.82 & -0.21 & -0.74 & -0.23 & -0.71 & -0.12 & -0.80 & -0.28 & -0.49 & 0.01 & -0.88 & -0.04 & -0.90 & -0.09 \\
\hline True colour & -0.80 & -0.18 & -0.72 & -0.21 & -0.71 & -0.11 & -0.78 & -0.26 & -0.42 & 0.00 & -0.82 & -0.01 & -0.87 & -0.05 \\
\hline Turbidity & -1.04 & -0.42 & -0.93 & -0.49 & -0.76 & -0.11 & -0.93 & -0.45 & -1.22 & 0.07 & -1.46 & -0.39 & -1.16 & -0.57 \\
\hline TS & 2.42 & 3.12 & 2.18 & 3.93 & -0.01 & -0.13 & 1.86 & 3.32 & 4.25 & -2.56 & 5.84 & 5.53 & 1.98 & 5.36 \\
\hline TSS & 1.27 & 2.06 & 1.21 & 2.66 & -0.21 & -0.14 & 0.77 & 2.44 & 1.85 & -2.22 & 3.49 & 4.06 & 0.50 & 3.14 \\
\hline $\mathrm{pH}$ & -1.05 & -0.43 & -0.95 & -0.51 & -0.77 & -0.11 & -0.99 & -0.46 & -1.33 & 0.08 & -1.52 & -0.43 & -1.24 & -0.64 \\
\hline Conductivity & 0.85 & 1.30 & 0.64 & 1.59 & -0.43 & -0.10 & 0.76 & 1.03 & 2.58 & -0.49 & 2.29 & 2.03 & 1.21 & 3.01 \\
\hline TDS & 0.08 & 0.60 & 0.00 & 0.74 & -0.57 & -0.11 & 0.06 & 0.42 & 0.99 & -0.26 & 0.75 & 1.03 & 0.22 & 1.53 \\
\hline Hardness & -0.69 & -0.12 & -0.60 & -0.05 & -0.70 & -0.12 & -0.75 & -0.22 & -0.41 & -0.03 & -1.58 & -0.47 & -1.27 & -0.69 \\
\hline DO & -1.06 & -0.45 & -0.96 & -0.52 & -0.77 & -0.11 & -1.00 & -0.48 & -1.34 & 0.08 & -1.54 & -0.45 & -1.25 & -0.67 \\
\hline BOD & -1.06 & -0.45 & -0.96 & -0.52 & -0.77 & -0.11 & -1.00 & -0.47 & -1.37 & 0.08 & -1.55 & -0.45 & -1.25 & -0.67 \\
\hline Calcium & -0.93 & -0.33 & -0.83 & -0.35 & -0.74 & -0.12 & -0.92 & -0.38 & -1.05 & 0.05 & -1.26 & -0.26 & -1.09 & -0.39 \\
\hline Magnesium & -1.05 & -0.45 & -0.96 & -0.52 & -0.77 & -0.11 & -0.99 & -0.47 & -1.36 & 0.07 & -1.53 & -0.45 & -1.25 & -0.68 \\
\hline Sulphate & -1.04 & -0.43 & -0.95 & -0.50 & -0.77 & -0.11 & -0.98 & -0.45 & -1.31 & 0.07 & -1.50 & -0.42 & -1.23 & -0.63 \\
\hline nitrate-nitrogen & -1.04 & -0.42 & -0.94 & -0.49 & -0.76 & -0.11 & -0.98 & -0.46 & -1.29 & 0.06 & -1.58 & -0.47 & -1.27 & -0.69 \\
\hline THBC & 8.40 & -1.92 & 7.52 & -2.34 & 7.69 & -1.56 & 8.51 & -1.56 & 5.05 & 3.30 & -1.58 & -0.47 & -1.27 & -0.69 \\
\hline TCBC & -0.12 & -0.13 & -0.60 & -0.54 & 2.44 & 4.89 & 0.54 & -0.73 & -1.41 & 0.09 & -1.58 & -0.46 & -1.27 & -0.69 \\
\hline THFC & -0.34 & -0.38 & 0.42 & -0.79 & 0.82 & -1.36 & -0.49 & 0.27 & 0.55 & 1.47 & -1.58 & -0.47 & -1.27 & -0.69 \\
\hline
\end{tabular}

recorded in station $\mathrm{A}$. Lowest mean water temperature $\left(26.12^{\circ} \mathrm{C} \pm 1.21^{\circ} \mathrm{C}\right)$ was recorded in station $\mathrm{C}$ while the height mean $\left(26.92^{\circ} \mathrm{C} \pm 1.11^{\circ} \mathrm{C}\right)$ was observed at station $\mathrm{D}$. The highest mean value for TS was recorded in station A while TSS, calcium, nitrate-nitrogen and hardness were highest in station B. Sulphate, BOD, cadmium, THBC, TCBC and THFC have maximum mean values at station $\mathrm{C}$ while highest mean values for water temperature, magnesium, TDS, turbidity and conductivity values were recorded at station D. Air temperature, DO, apparent colour and $\mathrm{pH}$ were highest in station $\mathrm{E}$. The highest mean value for THBC was observed at point $\mathrm{C}\left(2.22 \times 10^{4} \pm 1.63 \times 10^{4} \mathrm{CFU} \cdot \mathrm{mL}^{-1}\right)$, while the lowest mean THBC occurred at Point E $\left(1.84 \times 10^{3} \pm 2.44 \times 10^{3} \mathrm{CFU} \cdot \mathrm{mL}^{-1}\right)$ and 
there was no significant difference $(\mathrm{p}>0.05)$ between the sampling points. The highest mean value for TCBC was recorded at point $\mathrm{C}\left(1.19 \times 10^{4} \pm 1.56 \times 10^{4}\right.$ $\mathrm{CFU} \cdot \mathrm{mL}^{-1}$ ), while zero TCBC was recorded in point $\mathrm{E}$ and there was no significant difference $(p>0.05)$ between the points. The highest mean value for total heterotrophic fungi was recorded at point $\mathrm{C}\left(5.92 \times 10^{3} \pm 1.23 \times 10^{4} \mathrm{SFU} \cdot \mathrm{mL}^{-1}\right)$, while the lowest mean value occurred at point $\mathrm{D}\left(3.80 \times 10^{3} \pm 6.64 \times 10^{3}\right.$ SFU $\left.\cdot \mathrm{mL}^{-1}\right)$ and there was no significant difference $(\mathrm{p}>0.05)$ between the points.

Table 3 shows the results for monthly variations of the physico-chemical parameters measured. The highest mean value for air temperature $\left(28.8^{\circ} \mathrm{C} \pm\right.$ $0.27^{\circ} \mathrm{C}$ ) was recorded in April while the lowest value was recorded in October, and there was a highly significant difference $(\mathrm{p}<0.001)$ in monthly variation of air temperature for the study. The lowest mean water temperature $\left(25.44^{\circ} \mathrm{C} \pm\right.$ $\left.0.38^{\circ} \mathrm{C}\right)$ was recorded in September while the highest temperature $\left(28.80^{\circ} \mathrm{C} \pm\right.$ $\left.0.27^{\circ} \mathrm{C}\right)$ was recorded April with a significant difference $(\mathrm{p}<0.001)$ in the monthly variation. Significant difference $(\mathrm{p}<0.05)$ was recorded in the monthly values for apparent colour and true colour respectively. The highest mean value for TSS was recorded in April while there was significant difference $(p<0.01)$ in the monthly variation for TS in this study. Maximum mean concentration for DO was observed in September $(6.28 \pm 1.14 \mathrm{mg} / \mathrm{L})$ and there is significant difference $(\mathrm{p}<0.01)$ in monthly variation. BOD and $\mathrm{pH}$ mean concentration was highest in January and there was a significant difference $(\mathrm{p}<0.01)$ in the monthly variation for BOD. Sulphate concentration was highest in October and there was significant difference $(\mathrm{p}<0.05)$ in the monthly variation. Monthly concentration ranged recorded for $\mathrm{Mn}$ and $\mathrm{Pb}$ were higher in April (0.05- $0.11 \mathrm{mg} / \mathrm{L}$ and $0.02-$ $0.03 \mathrm{mg} / \mathrm{L})$ and there was highly significant difference $(\mathrm{p}<0.001)$ in monthly variation. The mean values for THBC, TCBC and THFC $\left(2.02 \times 10^{6} \pm 2.18 \times 10^{6}\right.$ $\mathrm{cfu} / \mathrm{ml}, 3.31 \times 10^{5} \pm 9.75 \times 10^{5} \mathrm{cfu} / \mathrm{ml}$, and $\left.4.91 \times 10^{5} \pm 1.05 \times 10^{6} \mathrm{cfu} / \mathrm{ml}\right)$ during the sampling period were extremely high, especially during the wet season than dry season.

\section{Principal Component Analysis (PCA) and Cluster Diagrams of Physico-Chemical Parameters of Olosuru Stream, Ikire Southwestern Nigeria}

The first two components for the wet season accounted for $91.64 \%$ of the cumulative variance. The first component accounted for $69.18 \%$ of the explained variance. TS and TSS recorded high positive loadings of 5.84 and 3.49, respectively while TDS recorded a positive loading of 0.75 (Table 6). The first two components for the dry season accounted for $84.73 \%$ of the cumulative variance. The first component accounted for $67.68 \%$ of the explained variance. Only TS recorded high load of 1.98 while TDS and TSS recorded a positive loading of 0.22 and 0.55 . The second component accounted for $17.05 \%$ of the explained variance. TS, TDS and TSS recorded high positive loadings of 5.36, 1.53 and 3.14 respectively. The first two components for station A accounted for $98.97 \%$ of the cumulative variance. The first component accounted for $79.94 \%$ of the explained 
variance. TS, TSS, conductivity and THBC recorded high positive loadings of $2.42,1.27,0.85$ and 8.40 respectively while TDS recorded a low positive loading of 0.08 . The second component accounted for $19.03 \%$ of the explained variance and TS, TDS, TSS and conductivity recorded a high positive loading of 3.12, $0.60,2.06$ and 1.30 respectively. The first two components for station B accounted for $94.87 \%$ of the cumulative variance. The first component accounted for $64.72 \%$ of the explained variance. TS, TSS and THBC recorded high positive loadings of 2.18, 1.21 and 7.52 respectively. The second component accounted for $30.15 \%$ of the explained variance and TS, TDS, TSS and conductivity recorded a high positive loading of 3.93, 0.74, 2.66 and 1.59 respectively. The first component for station $\mathrm{C}$ accounted for $64.55 \%$ of the explained variance while THBC, TCBC and THFC recorded high positive loadings of 7.69, 2.44 and 0.84 respectively. The first two components for station D accounted for $98.38 \%$ of the cumulative variance. The first component accounted for $77.84 \%$ of the explained variance. TS, TSS, conductivity, THBC and TCBC recorded high positive loadings of $1.86,0.77,0.76,8.51$ and 0.54 respectively while TDS recorded a low positive loading of 0.06 . The second component accounted for $20.54 \%$ of the explained variance and TS, TDS, TSS and THFC conductivity recorded a high positive loading of 3.32, $0.42,2.44,1.03$ and 0.27 respectively. The first component for station D accounted for $64.37 \%$ of the explained variance while TS, TDS, TSS, conductivity, THBC and THFC recorded high positive loadings of 4.25, $0.99,1.85,2.58,5.05$ and 0.55 respectively. Clustered diagram showing the relationship between the physico-chemical parameters and the stations. Figure 2 showing 4 major clustering diagram was formed: 1) Magnesium, true colour, Cd, $\mathrm{Mn}, \mathrm{Pb}, \mathrm{As}, \mathrm{TCBC}$ and Fe; 2) turbidity, TDS, conductivity, TSS, TS; 3) pH, nitrate-nitrogen, calcium, hardness water temperature and air temperature; 4) Magnesium, THBC, DO, apparent colour, BOD and THFC. Figure 3 showing one major clustered diagram were formed among the stations which are: station $\mathrm{D}$, station $B$, station A, station $\mathrm{E}$ and only station $\mathrm{C}$ was separate from others.

\section{Discussion}

The physico-chemical parameters in the water body vary in concentration along spatial, seasonal and monthly variation of Olosuru stream. The patterns of spatial distribution of physico-chemical parameters measured for the stream were generally similar except for calcium and hardness which showed significant difference for the five stations. Temperature is one of most important factor that influences primary production in any aquatic environment and It depends on the climate, sunlight and depth [19] [20] [21]. The mean of air temperature values, although higher, followed closely the changes in water temperatures and this might be attributed to the sampling time and climatic factor. Air and water temperature showed a strong positive relationship for the Olosuru stream, a similar report was documented by [22]. Lower mean temperature recorded during October and January was attributed to the effect of harmattan wind while the highest mean temperature recorded during April was attributed to the beginning 


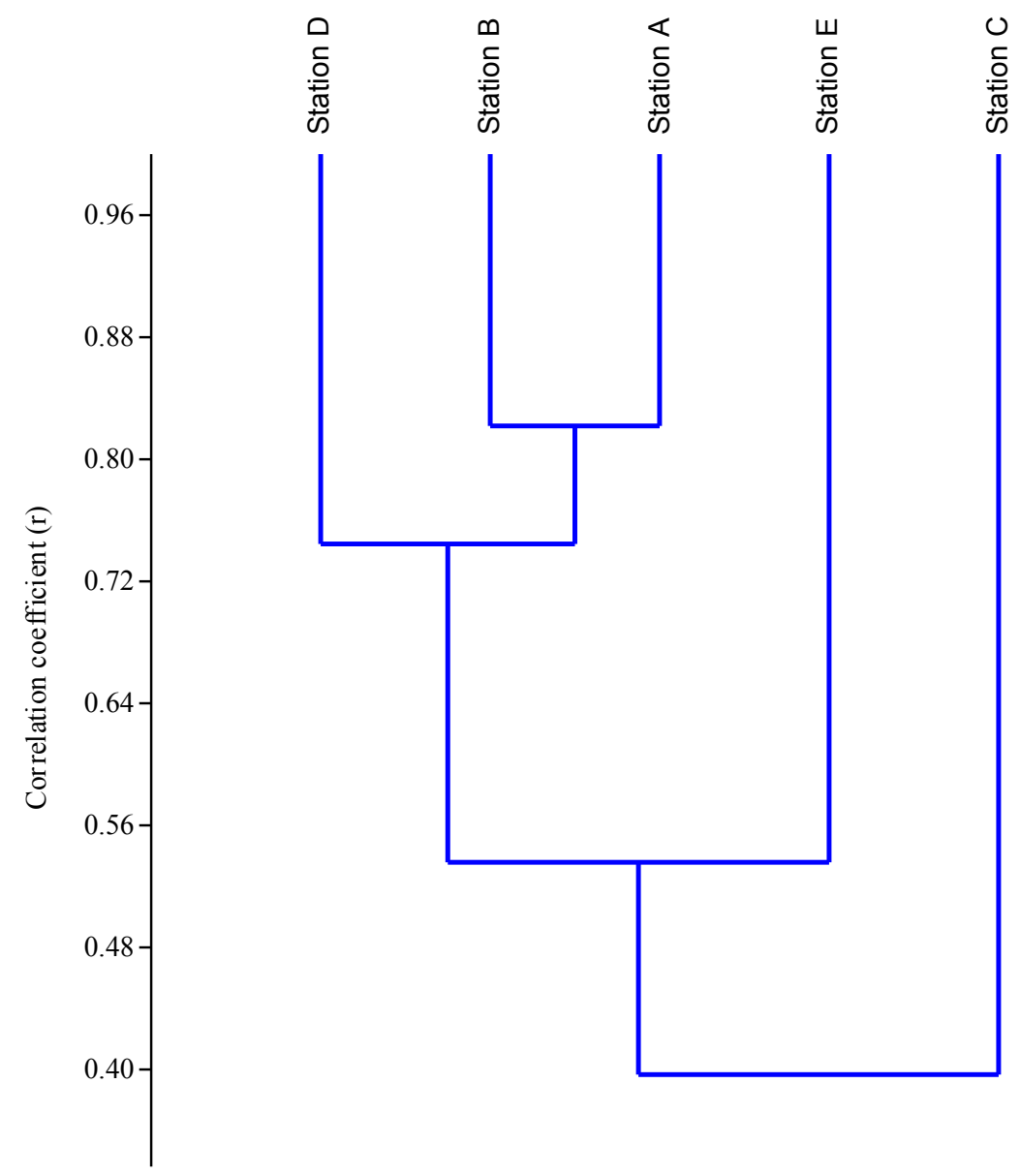

Figure 2. Cluster diagram showing relationship between the stations of Olosuru stream, Ikire Southwestern, Nigeria.

of the wet season when insolation was at its highest while similar reports have been observed for Owena Reservoir [23], Oyan and Asejire Lakes [24]. The minimum and maximum temperatures $\left(25.00\right.$ and $29.5^{\circ} \mathrm{C}$, respectively) are normal for tropical waters and are required for the normal growth of aquatic organisms. An overall $\mathrm{pH}$ ranged of $7.1-8.24$ with mean concentration (7.45 \pm 0.24 ) was observed for Olosuru stream and it is regarded as slightly alkaline. This is in agreement with the work of [25] who suggested that discharge of poultry wastes into stream water bodies increases the presence of lime-like materials such as calcium and magnesium in such stream. [26] recorded $\mathrm{pH}$ values ranging from 6.9 - 9.6, while [24] reported a $\mathrm{pH}$ range of 6.2 - 8.5. Accumulation of free carbon dioxide due to little photosynthetic activities of phytoplankton will lower the $\mathrm{pH}$ value of the water while intense photosynthetic activities of phytoplankton will reduce the free carbon dioxide content resulting in increased $\mathrm{pH}$ values [20]. There was a sharp decrease in the $\mathrm{pH}$ mean of Olosuru stream in September. This can be attributed to the increased organic matter brought in by rain as a result of runoff during peak wet season that tends to reduce dissolved oxygen through utilization of organic dehydration giving rise to a fall in $\mathrm{pH}$ [27]. 


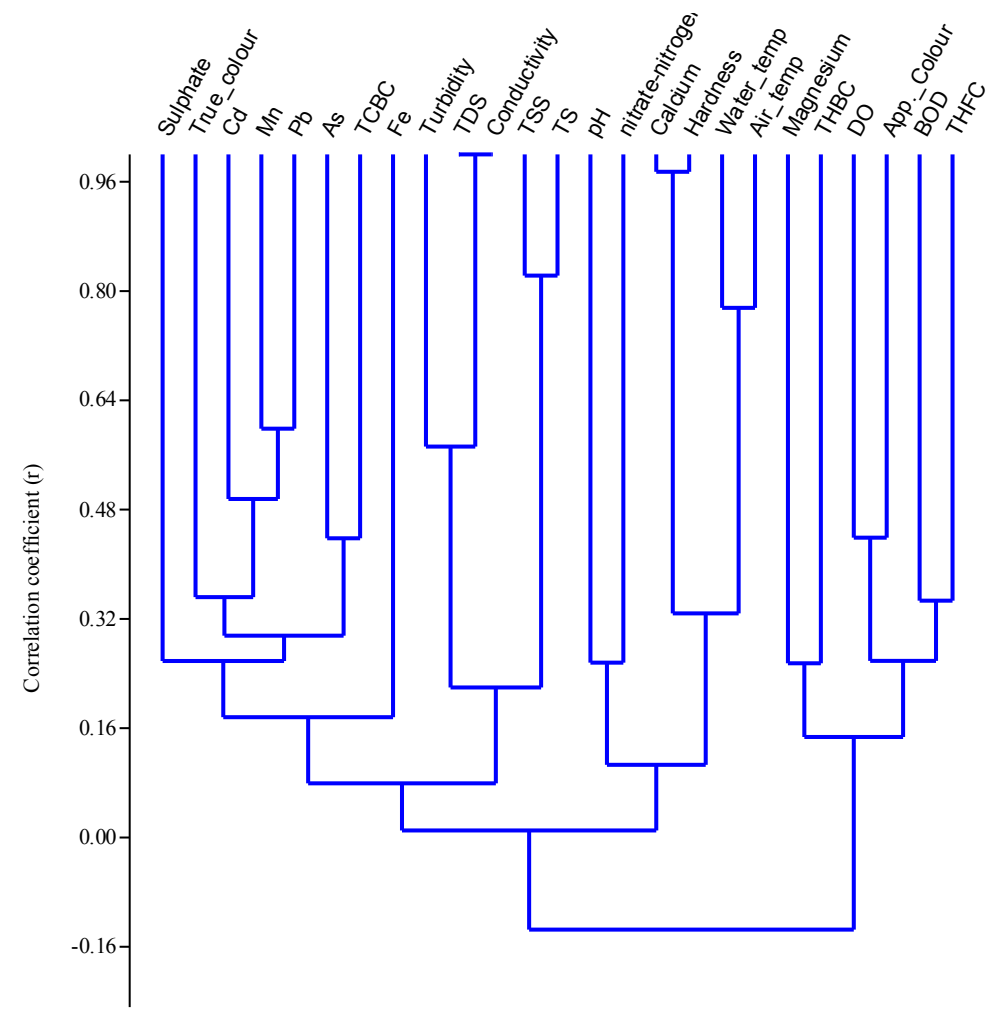

Figure 3. Cluster diagram showing relationship between the physico-chemical parameters and microbial loads of Olosuru stream, Ikire Southwestern, Nigeria.

Mean $\mathrm{pH}$ values were low in samples taken in station B before the point of waste discharge into the stream while the mean $\mathrm{pH}$ recorded for this study start to increase from the point of discharged. The higher mean values of $\mathrm{pH}$ observed in the point of discharged maybe linked to the influence of poultry waste discharges into the stream based on the presence of lime-like materials such as calcium and magnesium. Poultry manure contains about 100 pounds of calcium per ton on a dry weight basis [28]. According to the South African Department of Water Affairs and Forests [29], high pH in water could alter the toxicity of other pollutants, such as ammonia. The mean $\mathrm{pH}$ values of the stream are within the international permissible standard of a $\mathrm{pH}$ between 6.5 and 8.5 for drinking water [30] [31]. The overall turbidity ranged from 0.37 to $91.84 \mathrm{NTU}$ with mean values of $21.38 \pm 21.24 \mathrm{NTU}$ recorded for Olosuru stream. The highest mean turbidity value of $30.86 \pm 32.25 \mathrm{NTU}$ was observed in a sample collected at station $\mathrm{D}$ after poultry farm discharge point may indicated the presence of high concentrations of suspended materials, algae, and aquatic microscopic organisms [32]. However, high mean turbidity values between station C and D maybe as a result of poultry waste discharge [33]. Turbidity values exceeded the World Health Organization [31] standard of 5.0 NTU in drinking water. High turbidity is indicated by a change in water color and by a reduction of dissolved oxygen [34]. Because turbidity shelters bacteria, it is related to potential health effects 
[35]. The conductivity range (270.01 to $1546.98 \mu \mathrm{S} / \mathrm{cm})$ and mean value $(628.69$ $\pm 255.95 \mu \mathrm{S} / \mathrm{cm}$ ) for Olosuru stream during the study can be regarded as intermediate to high according to the classification by [36]. Conductivity levels below $50 \mu \mathrm{S} / \mathrm{cm}$ are regarded as low; those between $50-600 \mu \mathrm{S} / \mathrm{cm}$ are medium while those above $600 \mu \mathrm{S} / \mathrm{cm}$ are high conductivity levels. For many Nigerian inland water bodies the conductivities are much less than $500 \mu \mathrm{S} / \mathrm{cm}$ at the peak of the dry season and much less than $100 \mu \mathrm{S} / \mathrm{cm}$ during the rainy season [37]. The general trend in this study was that mean conductivity tended to slight decrease in the dry season compared with the wet season. Increased conductivities could result from low precipitation, higher atmospheric temperatures resulting in higher evapotranspiration rates and higher total ionic concentration, and saline intrusions from underground sources. It could also be due to a high rate of decomposition and mineralisation by microbes and nutrient regeneration from bottom sediments [37]. The significantly higher conductivity value recorded for Olosuru stream during the wet season suggests that allochthonous materials brought in by streams draining the catchments area plays a major role in the limnology studies of waterbody. The lower conductivity values recorded for the dry season may be due to the utilization of such allochthonous materials by the phytoplanktonic organisms of the reservoir [38]. In this study, True colour, TS, TSS, TDS, Hardness, calcium, magnesium, sulphate, $\mathrm{Fe}$ and $\mathrm{Mn}$ were significantly higher in mean values during wet season than dry season. This could be due to run-off entering the water body during the wet season period and reported three major components influencing the physico-chemistry of Olosuru stream which are trace metals, dissolved oxygen and ionic composition. The DO concentration ranged from 1.2 to $7.80 \mathrm{mg} / \mathrm{L}$ with overall mean value of $4.00 \pm$ $2.10 \mathrm{mg} / \mathrm{L}$. Low mean concentration of dissolved oxygen (DO) was observed during the dry season while high mean concentration of DO was observed during the wet season. The mean results of DO recorded during both season were within the minimum value of 5.0 milligrams per liter $(\mathrm{mg} / \mathrm{L})$ of DO standard for surface water, but DO values recorded for station E during November and September sampling periods were above standard. This shows correlations with the works of [39] [40]. The impact of the poultry waste discharges has a significant effect on the DO values of the stream. This conforms to the findings of [41]. The overall DO mean value obtained during the period of study, despite the expected pollution from the poultry waste discharge, could be attributed to aeration from the high stream flow rate at the time when the samples were collected [24]. The low DO value may be attributable to the drying effect and the depletion of oxygen by microorganisms from the poultry wastes [42]. The values of BOD were higher during the dry season than the wet season while the mean concentration of BOD observed at station C, November and January were generally greater than the BOD standard of $4.0 \mathrm{mg} / \mathrm{L}$ for drinking water, which relates to the work of [43]. Further, the study's BOD results were within standard limits of the Federal Environmental Protection Agency [44] standard of $6.0 \mathrm{mg} / \mathrm{L}$, indicating a 
low level of pollution in the stream from the poultry wastes discharge. Decaying riparian vegetation also might have added to the high values of BOD recorded during this study, especially in regard to the samples collected at the discharge point [45].

Nitrate-nitrogen $\left(\mathrm{NO}_{3}-\mathrm{N}\right)$ values ranged from 6.22 to $15.26 \mathrm{mg} / \mathrm{L}$ during the wet season and 3.67 to $16.09 \mathrm{mg} / \mathrm{L}$ during the dry season. In an unpolluted river, nitrate is usually less than $1.0 \mathrm{mg} / \mathrm{L}$ [46]. The mean concentration of $\mathrm{NO}_{3}-\mathrm{N}$ recorded during this study was above $10 \mathrm{~m} / \mathrm{L}$ expected for station $\mathrm{A}$ and in the month of February. This may be as result of poultry waste discharge and other anthropogenic activities, such as farming and the introduction of organic materials by runoff [47] [48]. The elevated $\mathrm{NO}_{3}-\mathrm{N}$ concentration indicates deterioration of the stream's water quality. Nitrate concentrations exceeding $10 \mathrm{mg} / \mathrm{L}$ are considered harmful and can cause methemoglobinemia in infants under six months as well as other health effects, such as diarrhea and respiratory diseases [49]. Studies have also revealed that long-term exposure to nitrate levels between 11 and $61 \mathrm{mg} / \mathrm{L}$ could increase the risk of hyperthyroidism [50], while nitrate levels greater than $25 \mathrm{mg} / \mathrm{L}$ have been associated with insulin dependent diabetes [51]. The $\mathrm{Pb}$ concentration observed in Olosuru stream ranged from 0.01 to 0.03 $\mathrm{mg} / \mathrm{L}$ with mean value of $0.02 \pm 0.01 \mathrm{mg} / \mathrm{L}$ while the concentrations of lead ranged from 0.01 to $0.03 \mathrm{mg} / \mathrm{L}$ in samples collected during the wet season and from 0.01 to $0.02 \mathrm{mg} / \mathrm{L}$ during the dry season. The mean values were higher during the wet season compared to the dry season. The presence of lead in the stream maybe traced to the deposition of lead-containing substances from nearby refuse sites, which have been carried into the stream via erosion, and from dry deposition from vehicular emissions [52] [53] [54]. At station A, the mean lead value is higher than the WHO permissible standards of $0.01 \mathrm{mg} / \mathrm{L}$. Lead is a toxic metal that is particularly harmful to children [55]. It affects the central and peripheral nervous system, organs, bones, and kidneys [35]. Cadmium concentrations in the stream were generally low during the study period, with values ranging from not detected 0.01 to $0.03 \mathrm{mg} / \mathrm{L}$ and highest mean concentration recorded during the wet and dry seasons, respectively. All the cadmium mean concentrations recorded were less than the $0.005 \mathrm{mg} / \mathrm{L}$ standard in drinking water [56]. High concentrations of cadmium in water could lead to cancer and affect hormones and enzymes, which can lead to malformations, including renal damage [57] [58]. In this study, bacterial species isolated can be grouped into thirteen genera namely Corynebacterium, Lactobacillus, Bacillus, Aeromonas, Pseudomonas, Yersinia, Morganella, Escherichia, Proteus, Vibrio, Micrococcus, Staphylococcus and Moraxella. The presence of Pseudomonas aeruginosa and other genera of Enterobacteriaceae (coliforms) such as Escherichia coli (faecal coliform), is an indication that the stream water was of poor microbiological quality. This agrees with the submission of [59] that the presence of E. coli in water samples is an indication of faecal contamination of the waterbody. The mean value of total coliforms from Olosuru stream exceeded the permissible World Health Organization standard which states that total coliform and faecal 
coliforms must not be detectable in any $100 \mathrm{ml}$ of water sample intended for drinking [60]. The presence of Pseudomonas aeruginosa and other genera of Enterobacteriaceae are associated with gastroenteritis [61] [62]. The Staphylococcus spp. is known to produce enterotoxin, which is harmful to human [63]. Twelve fungal species belonging to six genera were isolated in this study. These included Aspergillus fumigatus, Aspergillus parasiticus, Aspergillus brevipes, Rhizopus sp, Aspergillus flavus, Aspergillus niger, Mucor circinellioides, Mucor luteus, Rhizoctonia sp., Rhizopus stolonifers and Trichoderma harzianum. Some of these have been implicated in human chronic illnesses upon ingestion of contaminated water [64].

The dominance of Aspergillus spp. can be linked to the poultry wastes materials in the stream water. This was in agreement with the work of [65], who reported Aspergillus spp. as the dominant fungal isolate from poultry feeds obtained from commercial, self-compound and organized poultry feeds in Nigeria. Aspergillus flavus is known to produce aflatoxins which are the most toxic and potent hepatocarcinogenic natural compounds ever characterized [66]. A wide range of diseases in human could be caused by Aspergillus sp., ranging from hypersensitivity reactions to invasive infections associated with angioinvasion [66].

\section{Conclusion}

The study concluded that in comparison with international guide levels for drinking water, the water samples from Olosuru stream in Ikire were not potable and were unsuitable for drinking with respect to $\mathrm{pH}$, conductivity, TDS, sulphate, $\mathrm{BOD}_{5}$, cadmium, arsenic, manganese, total heterotrophic bacteria count, total coliform and total fungi. The result of this work also indicated a serious health hazard to the riparian users of water obtained from the stream without prior treatment.

\section{Acknowledgements}

The authors expressed their gratitude to the Laboratory of Limnology and Hydrobiology, Department of Zoology and the Department of Microbiology Central Laboratory, Faculty of Science, Obafemi Awolowo University, Ile-Ife, Nigeria for their permission to carry out this research work in their laboratories.

\section{Conflicts of Interest}

The authors declare no conflicts of interest regarding the publication of this paper.

\section{References}

[1] United Nations (2005) http://www.un.org/waterforlifedecade/background

[2] United States Geological Survey (USGS) (1987) National Water Summary 1987-Hydrologic Events and Water Supply and Use. USGS Water Supply Paper, 2350. 
[3] Shakhashiri, B. (2011) Chemical of the Week: Water. General Chemistry.

[4] Kumar, N. (1997) A View on Freshwater Environment. Ecological Environment and Conservation, 3, 3-4.

[5] USEPA (2001) EPA/Water Quality, Environmental Matters. Environmental Protection Agency, United States, 76.

[6] GESAMP (1988) Report of the Eighteenth Session, Paris 11-15 April 1988. GESAMP Reports and Studies No. 33. United Nations Educational, Scientific and Cultural Organization, Paris.

[7] Camargo Barros, G.S., Zen, S.D., Piedade Bacchi, M.R., Galvão de Miranda, S.H, Narrod, C. and Tiongco, M. (2003) Policy, Technical, Environmental Determinants and Implications of the Scaling-Up of Swine, Broiler, Layer and Milk Production in Brazil. Annex V. Final Report of IFPRI-FAO/Lead Livestock Industrialization Project: Phase II. International Food Policy Research Institute, Washington DC, 22.

[8] Gollehon, N., Caswell, M., Ribaudo, M., Kellogg, R., Lander, C. and Letson, D. (2001) Confined Animal Production and Manure Nutrients. Agriculture Information Bulletin No. 771. USDA, NRCS, ERS, Washington DC.

[9] Kumar, K., Gupta, S.C., Chander, Y. and Singh, A.K. (2005) Antibiotic Use in Agriculture and Its Impact on the Terrestrial Environment. Advances in Agronomy, 87, 1-54. https://doi.org/10.1016/S0065-2113(05)87001-4

[10] Young, J.L., Chang, M., Cochran, M.C. and Whiteside, L.L. (1996) Poultry Litter Land Application Rate Study. Final Report to Texas Natural Resources Conservation Commission. Stephen F. Austin State University and Angelina and Neches River Authority, 60.

[11] Cochran, M.C., Chang, M. and Young, J.L. (1998) Water Quality of Pastured East Texas Watersheds with Poultry Litter Applications. In: Potts, D.F. Ed., Rangeland Management and Water Resources, Proceedings of AWRA Special Conference, Reno, 27-29 May 1998, 457-466.

[12] McBroom, M.W., Chang, M. and Cochran, M.C. (1999) Water Quality Conditions of Streams Receiving Runoff from Land-Applied Poultry Litter in East Texas. Watershed Management to Protect Declining Species. In: Sakrison, R. and Sturtevant, P., Eds., AWRA's 1999 Annual Water Resources Conference, Seattle, 5-9 December 1999, 549-552.

[13] United States Environmental Protection Agency (USEPA) (2013) Literature Review of Contaminants in Livestock and Poultry Manure and Implications for Water Quality. Office of Water 4304T EPA 820-R-13_002, 18-137.

[14] National Population Commission (NPC) (2006) Nigeria Population Census Report. NPC, Abuja.

[15] Jones, H.A. and Hockey, R.D. (1964) The Geology of Part of Southwestern Nigeria: Explanation of 1: 250,000 Sheets Nos. 59 and 68. Authority of the Federal Government of Nigeria.

[16] Rahaman, M.A. (1975) Review of the Basement Geology of South-Western Nigeria. In: Kogbe, C.A., Ed., Geology of Nigeria, Rock View Limited, 538.

[17] Smyth, A.J. and Montgomery, R.F. (1962) Soils and Land Use in Central Western Nigeria. Soils and Land Use in Central Western Nigeria.

[18] Ademoroti, C.M.A. (1996) Standard Methods for Water and Effluents Analysis. Foludex Press Ltd., Ibadan, 182 p.

[19] Lewis, W.M. (2000) Basis for the Protection and Management of Tropical Lakes. Lakes and Reservoir Research and Management, 5, 35-48. 
https://doi.org/10.1046/j.1440-1770.2000.00091.x

[20] Gupta, S.K. and Gupta R.C. (2006) General and Applied Ichthyology (Fish and Fisheries) S. Chand and Company Ltd. Ram Nagar, New Delhi, 1130.

[21] Akinyemi, S.A. and Nwankwo, D.I. (2006) Biological Productivity of Two Reservoirs in Osun State, Nigeria. International Journal of Science and Technology Research, 3, 16-22.

[22] Adewale, T., Arowolo, T. and Iheoma, A. (2013) Evaluating the Environmental Impacts of Poultry Farming on Stream Water Quality: A Study from Abeokuta, Nigeria. Environmental Quality Management. http://www.researchgate.net/publication/264225416

[23] Oke, O.O. (1998) Plankton Diversity, Abundance and Productivity in the Owena Reservoir, Southwestern Nigeria. PhD Thesis, University of Ibadan, Ibadan.

[24] Ayoade, A.A., Fagade, S.O. and Adebisi, A.A. (2006) Dynamics of Limnological Features of Two Man-Made Lakes in Relation to Fish Production. African Journal of Biotechnology, 5, 1013-1021.

[25] Taiwo, A.M., Arowolo, T.A., Adekunle, I.M. and Adetunji, M.T. (2013) Evaluating the Environmental Impacts of Poultry Farming on Stream Water Quality: A Study from Abeokuta, Nigeria. Environmental Quality Management, 22, 79-93. https://doi.org/10.1002/tqem.21344

[26] Idowu, E.O. and Ugwumba, A.A.A. (2005) Physical, Chemical and Benthic Faunal Characteristics of a Southern Nigerian Reservoir. The Zoologist, 3, 15-25.

[27] Ogueri, C. (2004) Physico-Chemical Parameters, Fish Fauna and Fisheries of River Katsina-Ala, Nigeria. PhD Thesis, University of Ibadan, Ibadan.

[28] Zhang, H. (1998) Animal Manure Can Raise Soil pH. Production Technology, 10, 1-2.

[29] Department of Water Affairs and Forestry (DWAF) (1996) South African Water Quality Guidelines: Domestic Water Use. 2nd Edition, Author, Pretoria.

[30] US Environmental Protection Agency (US EPA) (1986) Quality Criteria for Water. Office of Water Regulation and Standards, Washington DC, USEPA-40015-86, 256 p.

[31] World Health Organization (WHO) (1993) Guidelines for Drinking Water Quality I. Recommendations. 2nd Edition, Author, Geneva.

[32] American Public Health Association (APHA) (1989) Standard Methods for the Examination of Water and Wastewater. 17th Edition, American Public Health Association, American Water Works Association and Water Pollution Control Federation, Washington DC.

[33] Nduka, J.K., Orisakwe, O.E. and Ezenkwe, L.O. (2008) Some Physicochemical Parameters of Potable Water Supply in Warri, Niger Delta Area of Nigeria. Scientific Research and Essay, 3, 547-551.

[34] Department of National Health and Welfare (Canada) (1978) Guidelines for Canadian Drinking Water Quality. Supporting Documentation, Author, Ottawa.

[35] Hassinger, E. and Watson, J. (1998) Health Effects of Drinking Water Contaminants. Arizona Water Series Number 5. University of Arizona, Cooperative Extension, Tucson.

[36] Adeleke, C.A. (1982) Studies on the Ecology and Feeding Habits of Lymnea natalensis (Krauss), Intermediate Host of Cattle Liver-Fluke in Ibadan Area. PhD Thesis, University of Ibadan, Ibadan. 
[37] Egborge, A.B.M. (1974) The Seasonal Variation and Distribution of Phytoplankton in the River Oshun, Nigeria. Freshwater Biology, 4, 177-191. https://doi.org/10.1111/j.1365-2427.1974.tb00088.x

[38] Oben, B.O. (2000) Limnological Assessment of the Impact of Agricultural and Domestic Effluent on Three Man-Made Lakes in Ibadan, Nigeria. PhD Thesis, University of Ibadan, Ibadan, 334 .

[39] Hodges, L. (1973) Environmental Pollution. A Survey of Emphasizing Physical and Chemical Principles. Holt, Rinehart and Winston, New York.

[40] Atobatele, O.E. and Ugwumba, O.A. (2018) Seasonal Variation in the Physico-Chemistry of a Small Tropical Reservoir AIBA Reservoir, Iwo, Osun, Nigeria. African Journal of Biotechnology, 7, 1962-1971. https://doi.org/10.5897/AJB2008.000-5043

[41] Ajayi, O.O., Kodaolu, E.O., Adeyemo, A.I. and Ogunsuyi, H.O. (2003) Effects of Poultry Droppings on the Physico-Chemical Properties of Soil and Water. Nigerian Journal of Soil Science, 13, 50-54.

[42] Izonfuo, L.W.A. and Bariweni, A.P. (2001) The Effect of Urban Runoff Water and Human Activities on Some Physico-Chemical Parameters of the Epie Creek in the Niger Delta. Journal of Applied Sciences and Environmental Management, 5, 47-55.

[43] Toms, R.G. (1975) Management of River Water Quality. In: Whitton, B.A., Ed., River Ecology, Studies in Ecology, Vol. 2, University of California Press, Berkeley, 538-564.

[44] Federal Environmental Protection Agency (FEPA) (1991) National Interim Guidelines and Standards for Environmental Pollution in Nigeria. Author, Abuja.

[45] Wilcock, R.J., McBride, G.B., Nagels, J.W. and Northcott, G.L. (1995) Water Quality in a Polluted Lowland Stream with Chronically Depressed Dissolved Oxygen: Causes and Effects. New Zealand Journal of Marine and Freshwater Research, 29, 277-288. https://doi.org/10.1080/00288330.1995.9516661

[46] Meybeck, M. (1982) Carbon, Nitrogen and Phosphorus Transport by World Rivers. American Journal of Science, 282, 401-450. https://doi.org/10.2475/ajs.282.4.401

[47] Adeyeye, E.I. and Abulude, F.O. (2004) Analytical Assessments of Some Surface and Groundwater Resources in Ile Ife, Nigeria. Journal of the Chemical Society of Nigeria, 29, 98-103.

[48] Aneja, V.P., Nelson, D.R., Roelle, P.A. and Walker, J.T. (2003) Agricultural Ammonia Emissions and Ammonium Concentrations Associated with Aerosols and Precipitation in the Southeast United States. Journal of Geophysical Research, 108. https://doi.org/10.1029/2002JD002271

[49] Ward, M.H., Dekok, T.M., Levallois, P., Brender, J., Gulis, G. and Nolan, B.T. (2005) Workgroup Report: Drinking Water Nitrate and Health-Recent Findings and Research Needs. Environmental Health Perspectives, 113, 1607-1614. https://doi.org/10.1289/ehp.8043

[50] Seffner, W. (1995) Natural Water Contents and Endemic Goiter. International Journal of Hygiene and Environmental Medicine, 196, 381-398.

[51] Kostraba, J.N., Gay, E.C., Rewera, M. and Hamman, R.F. (1992) Nitrate Levels in Community Drinking Waters and Risks of IDDM: An Ecological Analysis. Diabetes Care, 15, 1505-1508. https://doi.org/10.2337/diacare.15.11.1505

[52] Inanc, B., Kinaci, C., Sevimli, M.F., Arikan, O. and Ozturk, M. (1998) Pollution Prevention and Restoration in the Golden Horn of Istanbul. Water Science and Technology, 37, 129-136. https://doi.org/10.2166/wst.1998.0317 
[53] Martin, J.C., Hoggart, C. and Matissa, A. (1998) Improvement Priorities for Sewage Treatment in Latvian Small and Medium Sized Towns. Water Science and Technology, 37, 137-144. https://doi.org/10.2166/wst.1998.0318

[54] Inoue, T. and Ebise, S. (1999) Runoff Characteristics of COD, BOD, C, N, and P Loading from Rivers to Enclosed Coastal Seas. Marine Pollution Bulletin, 23, 11-14. https://doi.org/10.1016/0025-326X(91)90641-5

[55] Roberts, J.R. (1999) Metal Toxicity in Children. Training Manual on Pediatric Environmental Health: Putting It into Practice. Children's Environmental Health Network, Emeryville.

[56] US Environmental Protection Agency (USEPA) (2002) National Recommended Water Quality Criteria. 231. http://www.epa.gov/waterscience/pc/revcom.pdf

[57] Donalson, W.E. (1980) Trace Element Toxicity. In: Hodgson, E. and Guthrie, F.E., Eds., Introduction to Biochemical Toxicology, Elsevier, New York, 330-340.

[58] Lewis, R.J. (1991) Hazardous Chemicals Desk Reference. 2nd Edition, Van Nostrand Reinhold, New York.

[59] Bakare, A.A., Lateef, A., Amuda, O.S. and Afolabi, R. (2003) The Aquatic Toxicity and Characterization of Chemical and Microbiological Constituents of Water Samples from Oba River, Odo-oba, Nigeria. Asia Journal of Microbiology Biotechnology and Environmental Sciences, 5, 11-17.

[60] World Health Organization (WHO) (2007) Combating Waterborne Diseases at the Household Level. World Health Organization, Geneva.

[61] Zuane, J. (1996) Handbook of Drinking Water Quality. 2nd Edition, Van Nostrand Reinhold, New York. https://doi.org/10.1002/9780470172971

[62] Adeniyi, I.F. and Olabanji, I.O. (2005) The Physico-Chemical and Bacteriological Quality of Rainwater Collected over Different Roofing Materials in Ile-Ife, Southwestern Nigeria. Chemistry and Ecology, 21, 149-166.

https://doi.org/10.1080/02757540500117318

[63] Okonko, I.O., Ogunjobi, A.A., Adejoye, O.D., Ogunnusi, T.A. and Olasogba, M.C. (2008) Comparative Studies and Microbial Risk Assessment of Different Water Samples Used for Processing Frozen Sea-Foods in Ijora Olopa, Lagos State, Nigeria. African Journal of Biotechnology, 7, 2902-2907.

[64] Obire, O. and Putheti, R.R. (2009) Fungi in Bioremediation of Oil Polluted Environments. Sigma, 1-10.

[65] Young, S., Tiawo, A. and Dave, O. (2014) Mycological Quality of Commercially Self-Compound and Organized Poultry Feeds in Nigeria. International Journal of Mycology and Plant Pathology, 1, 45-48.

[66] Bennett, J.W. and Klich, M. (2003) Mycotoxins. Clinical Microbiology Review, 16, 497-516. https://doi.org/10.1128/CMR.16.3.497-516.2003 